\title{
Nearly free electron states in carbon nanotube bundles
}

\author{
Susumu Okada, ${ }^{1}$ Atsushi Oshiyama, ${ }^{2}$ and Susumu Saito ${ }^{3}$ \\ ${ }^{1}$ Institute of Material Science, University of Tsukuba, Tennodai, Tsukuba 305-8573, Japan \\ ${ }^{2}$ Institute of Physics, University of Tsukuba, Tennodai, Tsukuba 305-8571, Japan \\ ${ }^{3}$ Department of Physics, Tokyo Institute of Technology, 2-12-1 Oh-okayama, Meguro-ku, Tokyo 152-8551, Japan
}

(Received 22 November 1999)

\begin{abstract}
On the basis of density-functional theory, we study electronic structures of thin carbon nanotube bundles. We find that nearly-free-electron (NFE) states, which are distributed in intra- and intertube regions, are present at $3 \sim 4 \mathrm{eV}$ above the Fermi level. In the intertube region the NFE state of the nanotube bundles are strongly modified from that of the isolated nanotube. We also find that mixing of the NFE state with $s p^{2}$ orbitals at atomic sites is decisive in a peculiar behavior of their charge distribution. Importance of the NFE state in transport properties is discussed.
\end{abstract}

Bundles of single-wall carbon-nanotubes were recently synthesized using laser ablation technique, ${ }^{1}$ and manifest themselves as an example of hierarchical solids. The hierarchical solid here is characterized by its hierarchy in structure where atoms constitute intermediate units (a tube ${ }^{2}$ in this case) and then the units constitute a solid. Solid $\mathrm{C}_{60}{ }^{3,4}$ is another example of the hierarchical solid. The two-dimensional solid such as graphite may be regarded as a hierarchical solid since atoms constitute a layer and the layers constitute a solid there. Each unit (a tube, a fullerene, or a layer) of the hierarchical solids has its peculiar properties and the properties are modified in general by unit-unit interactions. To clarify uniqueness of the hierarchical solids, it is thus imperative to assess effects of the unit-unit interactions.

One of the characteristic features of the hierarchical solid is its unusual space contained inside. The space induces unique electron states: The space is large enough in some direction for an electron to behave as it does in vacuum; surrounding atoms are close enough, on the other hand, to induce negative potential for the electron. Consequently, nearly free electron (NFE) states may exist below the vacuum level. In fact, interlayer states of graphite ${ }^{5,6}$ and intercluster states of solid $\mathrm{C}_{60}{ }^{7}$ have been found to exist and play important roles in doping or intercalation of foreign atoms and molecules into the materials. Also in a single carbon nanotube ${ }^{8}$ and in a boron nitride nanotube, ${ }^{9}$ the calculations show the existence of a NFE state whose amplitude is inside the tube. We focus on the NFE states in the nanotube bundles here and explore how the NFE state keeps its characteristics in the bundles.

In this paper, we report first-principles calculations that clarify characteristics of NFE states in carbon nanotube bundles. We choose $(6,0),(12,0)$, and $(6,6)$ tubes as prototypes and optimize geometries of tube bundles. We find that the NFE state for each bundle is located below the vacuum level (at about 3 $4 \mathrm{eV}$ above the Fermi level), and that its amplitude is mainly in the intertube region and inside the tubes. Owing to the tube-tube interaction in the bundle, the intertube distribution of the NFE state is modulated from that of the isolated nanotube. In addition, we find that the NFE state has an amplitude also on the tubes where it has a character of $s$ and $p$ orbitals of carbon atoms. Importance of the NFE states in electronic-transport properties is discussed.
All calculations have been performed in the local-density approximation in the density-functional theory. ${ }^{10,11}$ To express the exchange-correlation potential of electrons, we use a functional form fitted to the Ceperley-Alder result. ${ }^{12,13}$ Norm-conserving pseudopotentials generated by using the Troullier-Martins scheme are adopted to describe the electron-ion interaction. ${ }^{14,15}$ In constructing the pseudopotentials, core radii adopted for $\mathrm{C} 2 s$ and $2 p$ states are both 1.5 bohrs. The valence wave functions are expanded by the plane-wave basis set with a cutoff energy of $50 \mathrm{Ry}$, which is known to give enough convergence of total energy to discuss the relative stability of various carbon phases. ${ }^{14} \mathrm{We}$ adopt the conjugate-gradient minimization scheme both for the self-consistent electronic-structure calculation and for the geometric optimization. ${ }^{16}$ Integration over Brillouin zone is carried out using 54-k-point sampling.

The tubes that we explore are metallic, $(6,6)$ (Refs. 17 and 18 ), or narrow-gap semiconducting, $(6,0)$ and (12,0) (Refs. 17 and 19), in their single-tube forms. We place the tubes in parallel in a two-dimensional hexagonal lattice in which a unit cell contains a single tube. The lattice constants in the direction perpendicular to the tubes, which are identical to distances between centers of adjacent nanotubes, are determined by the total-energy minimization: They are $7.9 \AA$, $11.4 \AA$, and $12.6 \AA$ for the $(6.0),(6,6)$, and $(12,0)$ bundles, respectively. Internal coordinates of carbon atoms in a cell are also fully optimized. Under the optimized structures, diameters of each tube are $4.73 \AA, 8.10 \AA$, and $9.28 \AA$ for the $(6,0),(6,6)$, and $(12,0)$ tubes, respectively. They are almost identical to the diameters of an isolated tube, i.e., $4.73 \AA$ for the isolated $(6,0)$ tube. Electronic band structures of the $(6,0)$-nanotube bundle, the $(12,0)$ bundle, and the $(6,6)$ bundle are shown in Fig. 1. All of these systems are found to be metallic. The isolated $(6,6)$ tube is metallic so that the bundle is also metallic. On the other hand, the intertube interaction is essential to render the semimetallic $(6,0)$ and $(12,0)$ tubes metallic in their bundle forms.

Overall features of the band structures of the nanotube bundles are qualitatively the same as those of corresponding isolated nanotubes that are obtained by the generalized tightbinding model. ${ }^{17} \mathrm{~A}$ new state that is absent in the tightbinding model, however, appears in each nanotube bundle. It is labeled ' $\alpha$ '" in Fig. 1. This state $\alpha$ has NFE character: The calculated effective mass along the tube direction is 

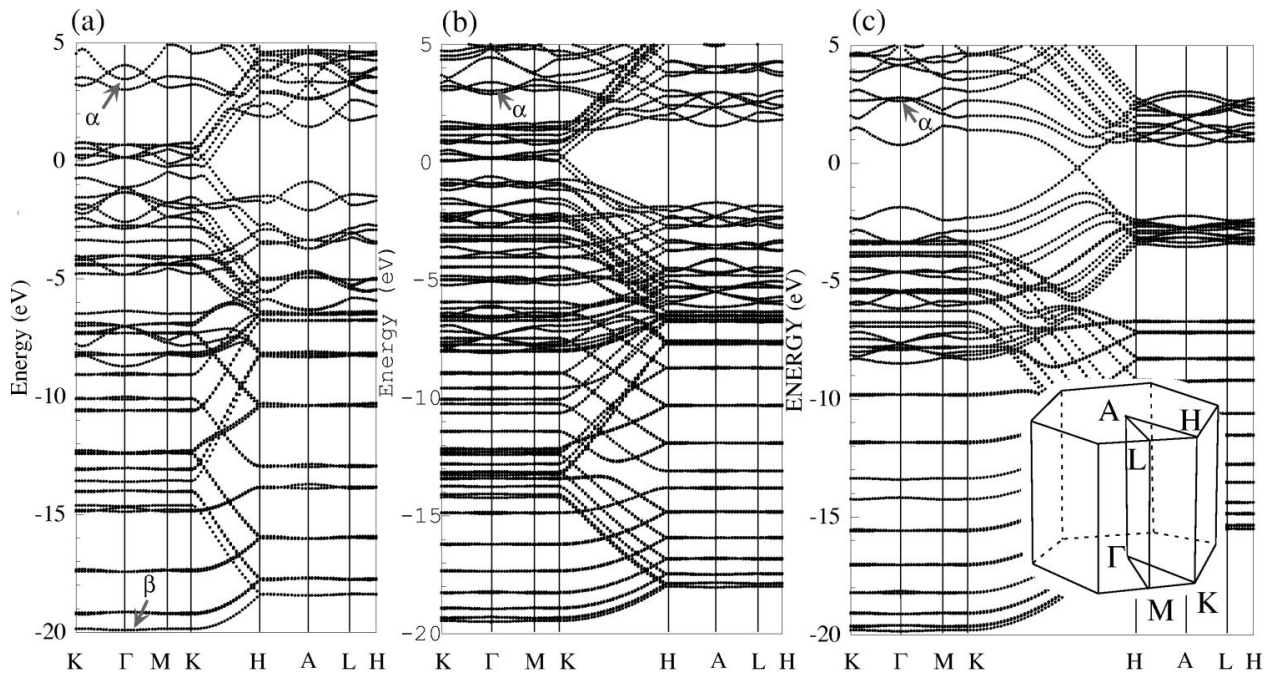

FIG. 1. Energy-band structures of (a) the $(6,0),(b)$ the $(12,0)$, and (c) the $(6,6)$ carbon nanotube bundles. The NFE states and their partners (see text) are indicated by ' $\alpha$ ' and ' $\beta$,' respectively. Energy is measured from the Fermi energy $E_{F}$. Inset (c): Symmetry points and lines in Brillouin zone of the hexagonal lattice.
$1.11 m_{0}$ and $1.07 m_{0}$ for the $(6,0)$ and $(6,6)$ nanotube bundles, respectively. The energy levels of the states $\alpha$ are located at $3.46 \mathrm{eV}, 3.00 \mathrm{eV}$, and $2.67 \mathrm{eV}$ above the Fermi level for the $(6,0),(12,0)$, and $(6,6)$ nanotube bundles, respectively. From the work function of graphite, the vacuum level is estimated to be about $5 \mathrm{eV}$ above the Fermi level. ${ }^{5}$ Hence the states $\alpha$ (the NFE states) are likely to be below the vacuum level.
Contour maps of the charge density of the state labeled $\alpha$ at the $\Gamma$ point unequivocally reveal its NFE character [Figs. 2(a) and 2(b)]. The eigenfunction of $\alpha$ is dominantly distributed within tubes. It is also distributed in regions between tubes: The peak value of the eigenfunction in the intertube region is a half of that in the intratube region. It is noteworthy that the highest density is at the center of each tube for (a)

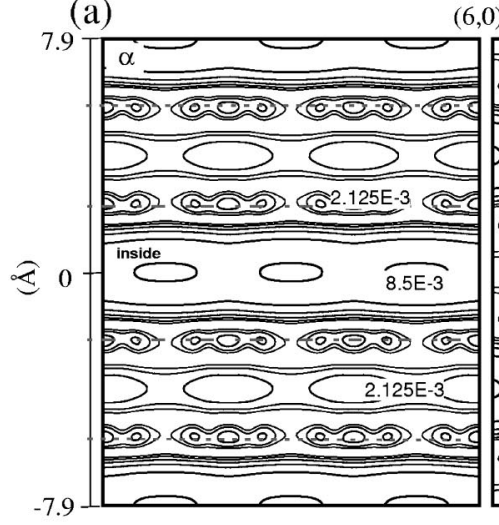

$(6,0)$

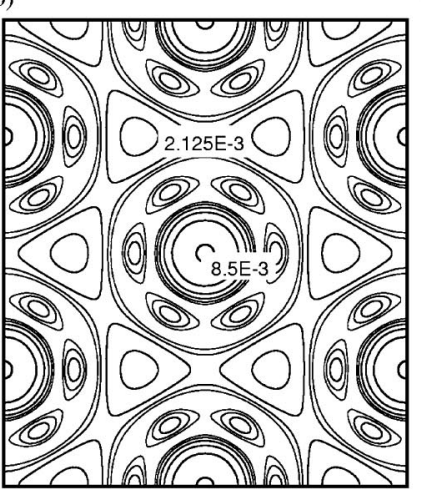

(b)

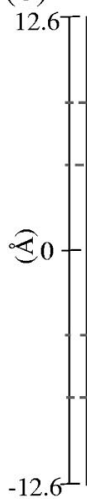

$(12,0)$

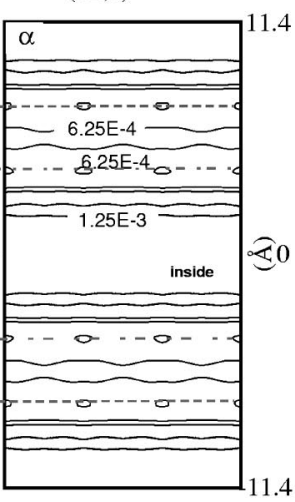

$(6,6)$

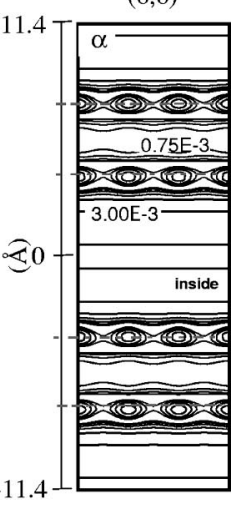

(c)
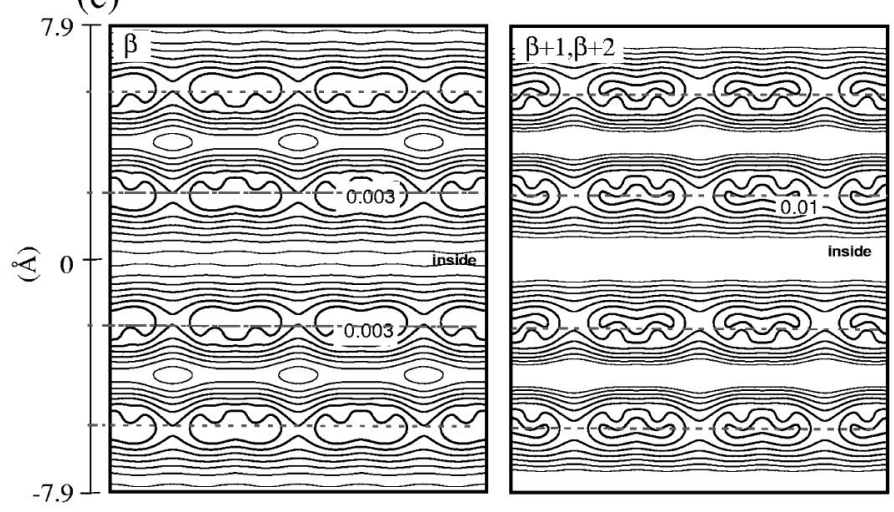

FIG. 2. Contour maps of the charge distribution of the NFE states (labeled $\alpha$ in Fig. 1) and the related at $\Gamma$ point. Each contour represents twice (or half) the density of the adjacent contour lines. The values shown in figures are in units of $e /$ (a.u.). ${ }^{3}$ (a) The NFE state $\alpha$ of the $(6,0)$ nanotube bundle represented on a cross section including tube axes (left panel) and on the other cross-section vertical to the tube axes (right panel). A pair of dot-dashed lines denote positions of walls of a nanotube, whereas dashed lines denote positions of walls of adjacent nanotubes. (b) The NFE state $\alpha$ of the $(12,0)$ bundle (left panel) and that of the $(6,6)$ bundle (right panel). (c) The partner state, labeled $\beta$ in Fig. 1(a), (left panel) and the sum of the two states near the $\beta$ (right panel) of the $(6,0)$ bundle, represented on a cross section including the tube axis. 

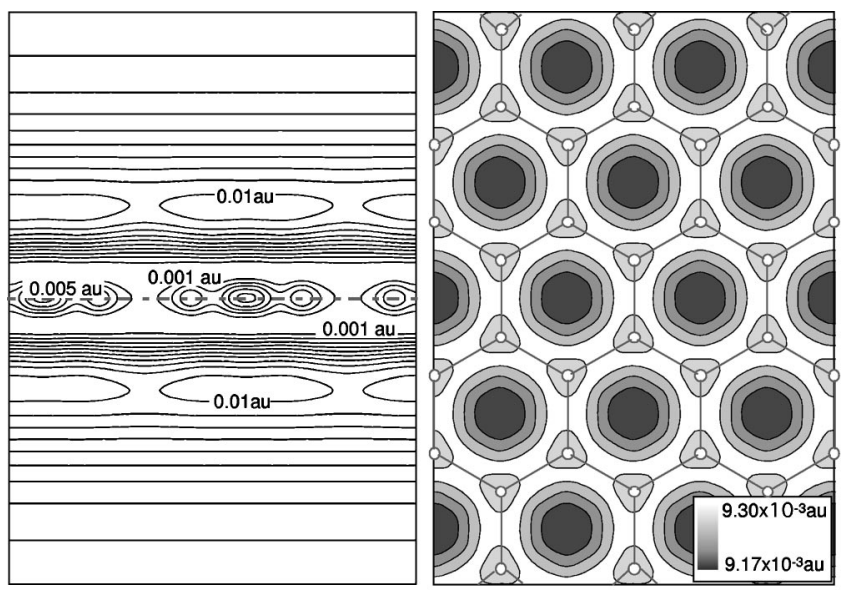

FIG. 3. Contour map of the NFE state at the $\Gamma$ point of graphite monolayer: (a) a side view and (b) a top view. Each contour represents twice (or half) the density of the adjacent contour lines. The values shown in figures are in units of $e /$ (a.u.). ${ }^{3}$ Dot-dashed lines in (a) denote the position of the graphite layer.

the $(6,0)$ tube bundle, whereas it is not at the center but along the inner circumference with the radius smaller than that of the nanotube circumference by about $2.2 \AA$ for the $(12,0)$ and $(6,6)$ tube bundles [Fig. 2(b)]. In any case, the state is unique in a sense that the charge density is substantially uniform along the tubes and distributed a little on carbon atoms. This behavior is similar to NFE states (interlayer state) of graphite. ${ }^{5,6}$ As is shown in Fig. 3, the highest amplitude of the eigenfunction of the NFE states of a graphite monolayer is located off the sheet by about $2.4 \AA$. Irrespective of the shape of carbon atom arrays, the state that has the NFE character has the highest amplitude at the region about $2 \AA$ off the atom arrays. In the case of the $(6,0)$ nanotube bundle, the radius of the tube is $2.3 \AA$ so that the density is highest at the center of the tube. For comparison, we calculate the charge density of the NFE state of an isolated $(6,0)$ nanotube (Fig. 4). As is expected, the charge density distribution is concentric. Again the highest density is at the center of the tube. Outside the tube, the density is highest on the outer circumference with the radius larger than that of the atomic circumference of nanotube by about $2.2 \AA$. The distribution of the charge density of the NFE state is modified in a region outside the tubes in the bundle forms. As is shown in Fig. 2(a), the distribution is no longer concentric in the intertube region and has a peak along the lines located at the centers of the three adjacent nanotubes. The distribution of the eigenfunction at this point is four times larger than that at the middle between two adjacent nanotubes. This is due to the hexagonal arrangement of nanotubes and the resulting intertube interactions. This opens a possibility of controlling the distribution of the charge density of the NFE state by certain arrangements of nanotubes.

It is noteworthy that the NFE state in Fig. 2 has its amplitude also at carbon atoms. The peak value of the eigenfunction around the carbon atoms is $1 / 8$ of the highest value within the tubes. The orbitals that constitute the NFE state around the carbon atoms are mainly $s p^{2}$ orbitals on the carbon sheet. The $p$ orbital perpendicular to the carbon sheet $\left(p_{\perp}\right)$ is also mixed due to the curvature of the tubes. We thus state that the NFE state has a character of $s p^{2+\delta}$ (Ref. 20) around carbon atoms. Analyses of the eigenfunction reveal that there are nodes between the atomic circumference and the outer circumference of nanotube where the density has a peak. Hence the NFE state we focus on has the antibonding character. This leads to a natural expectation that there is a bonding state below the Fermi level that has the $s p^{2+\delta}$ character around carbon atoms and the NFE character off the atom sheets. We explore valence bands and indeed find that the lowest-valence state, labeled $\beta$ in Fig. 1(a), has such character. Figure 2(c) shows the calculated charge densityof-state $\beta$ at $\Gamma$ point. The highest value of the charge density is on the $s p^{2+\delta}$ orbitals of carbon atoms but the density has another peak in the intertube region. For comparison we also plot the charge density of the nearby valence states in Fig. 2(c). It is clear that these states have amplitude neither within the tube nor in the intertube region. We also expect another NFE state located in higher energy, which has a character of the $p_{\perp}$ orbital around the carbon atoms. The NFE state is thus an ambivalent eigenstate: It is indeed NFE-like in the inter- and intratube regions and at the same time keeps carbon heritage on circumference of nanotubes.

As is stated above, the amplitude of the NFE state of the nanotube bundle is higher within the tubes than in the intertube region. This higher distribution inside is also observed for an isolated tube (Fig. 4). This behavior of the charge distribution is a result of the ambivalence of the NFE state combined with the curvature of the tubes. The $s p^{2+\delta}$ orbitals are bent along the circumference of the tube and thus has higher amplitudes within the tube compared with the outside. Therefore, to increase the mixing with $s p^{2+\delta}$ orbitals and hereby gain energies, the NFE state has higher amplitudes inside. This picture is confirmed by variation of the energy level of the NFE state. The values at the $\Gamma$ point of the $(6,0)$ and the $(12,0)$ nanotube bundles are $3.46 \mathrm{eV}$ and $3.00 \mathrm{eV}$, respectively, above the Fermi level. This is consistent with

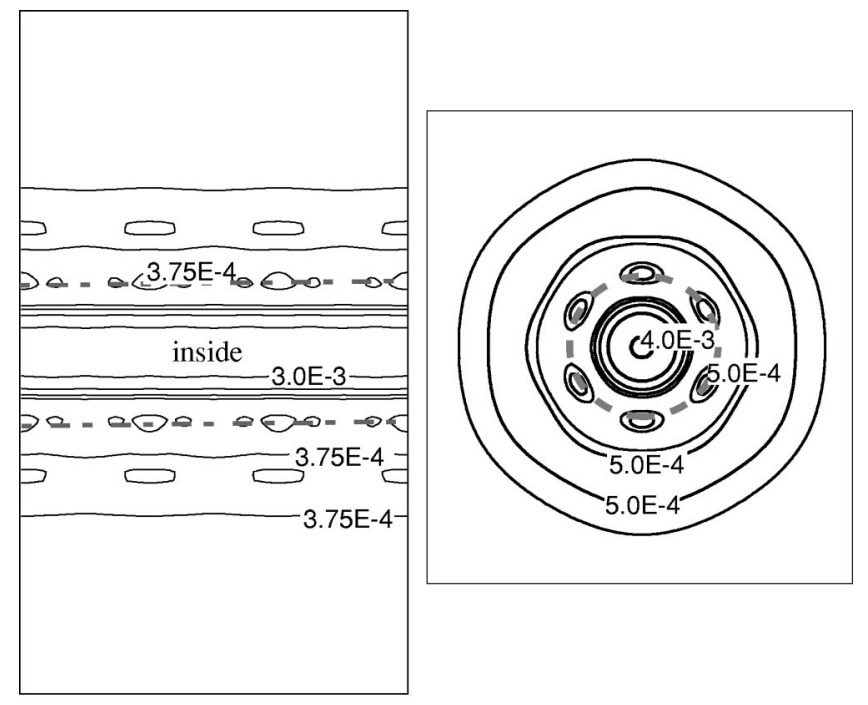

FIG. 4. Contour map of the charge distribution of the NFE state of the isolated $(6,0)$ nanotube at the $\Gamma$ point. A cross section including tube axes (left panel) and on the other cross section vertical to the tube axes (right panel). Each contour represents twice (or half) the density of the adjacent contour lines. The values shown in figures are in units of $e /$ (a.u.). ${ }^{3}$ A pair of dot-dashed lines denote positions of walls of a nanotube. 
the picture that thinner tubes induce more bending and thus more mixing, leading to an upward shift of the NFE state with the antibonding character. The mixing of the NFE and the $s p^{2+\delta}$ characters is already observed in in-plane distribution of the interlayer or the surface state of graphite (Fig. $3)$.

The NFE state is expected to have long transport relaxation time $\tau$ along the direction parallel to the tube axis since the amplitude of the eigenfunction is large in the region free from scatterers. The relaxation time of the state is, in general, evaluated by

$$
\frac{1}{\tau} \simeq \frac{2 \pi}{\hbar} \sum_{\mathbf{k}^{\prime}} \delta_{\varepsilon_{\mathbf{k}^{\prime}}, \varepsilon_{\mathbf{k}}}\left|\left\langle\psi_{\mathbf{k}^{\prime}}|U| \psi_{\mathbf{k}}\right\rangle\right|^{2} .
$$

Assuming $\mathrm{U}$ from short range scatterers located on the circumference of the tubes and using the calculated eigenfunctions, we evaluate a ratio of the relaxation time of the NFE state to that of the state near the Fermi level. The ratio is about 30.5 and 17.4 for the $(6,0)$ and the $(6,6)$ bundles, respectively. Therefore a peak in $I-V$ characteristics or in photoconductivity along the tube axis is expected when the NFE state becomes a channel of the conduction.

Doping or intercalation of foreign atoms are another way to fill the NFE state with electrons and generate highly conducting materials. When we adopt a rigid-band model, the number of required electrons in order to fill the NFE state can be calculated from the density-of-states (DOS) of the nanotube bundles. In Fig. 5, we show the DOS of the $(6,0)$ and the $(6,6)$ nanotube bundles, along with the Fermi-level positions expected upon electron doping. The Fermi level is shifted up to the NFE state upon doping of 12 and 10 electrons per unit cell for $(6,0)$ and $(6,6)$ bundles, respectively. The hybridization between the NFE state of the tube bundle and the state of foreign atoms could shift the NFE state downward, as in the case of alkali- or alkaline-earth-metal atom doping. ${ }^{8}$ Furthermore, as we discussed above, the NFE state is shifted downwards in energy by increasing the diameter of nanotubes. Hence it is expected that an actual number of doped electrons required for the highly conducting nanotube bundles may be smaller than 12 for thicker nanotube bundles.

Intercalation of atoms and molecules into nanotubes has been studied experimentally. ${ }^{21-23}$ The saturation composition is reported to be $A \mathrm{C}_{8}$ for $\mathrm{K}$ and Cs. ${ }^{21,22}$ In these compounds, intercalants may be mostly doped in the intertube regions owing to closed ends of nanotubes as well as the small sizes of pentagons and hexagons in the carbon network. However, intercalants should be stabilized more in the intratube regions than in the intertube regions due to the stronger hybridization expected. The spatial distribution of the NFE state is larger in the intratube regions than in the intertube regions.

In summary, we have studied the electronic band structures of thin carbon nanotube bundles, $(6,0),(6,6)$, and
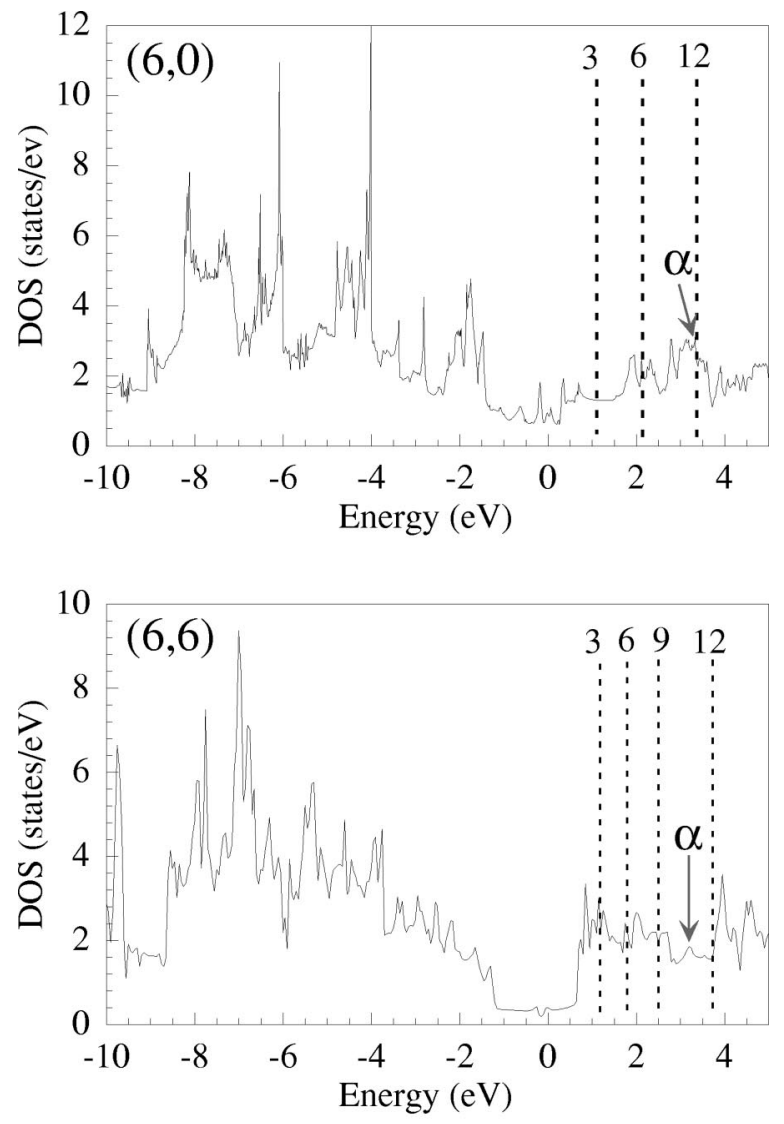

FIG. 5. Density-of-states for the (a) $(6,0)$ and (b) $(6,6)$ nanotube bundles. Energy is measured from the Fermi energy $E_{F}$ of pristine nanotube bundles. Fermi-level positions expected upon doping $n$ electrons per unit cell are shown by the dashed lines with the number $n$. The NFE state at the $\Gamma$ point is denoted by the " $\alpha$."

$(12,0)$, based on the local-density approximation in the density-functional theory. We have found the nearly free electron states below the vacuum level. The NFE states have amplitudes in the intratube and the intertube regions where atoms are absent. We also find that the NFE state has a character of $s p^{2+\delta}$ orbitals. It is clarified that owing to this mixing the largest amplitude of the NFE state is located in the intratube region. The NFE states are found to be of high importance in discussing the transport properties of doped carbon nanotube bundles.

Numerical calculations were performed on the Fujitsu VPP500 computer at Institute for Solid State Physics, University of Tokyo and the NEC SX3/34R computer at Institute for Molecular Science, Okazaki National Institute. This work was supported in part by JSPS under contract No. RFTF96P00203, The Nissan Science Foundation, and The Grant-in-Aid for Scientific Research No. 11740219 and the Priority Area "Fullerenes and Nanotubes" by the Ministry of Education, Science, and Culture of Japan.
${ }^{1}$ A. Thess, R. Lee, P. Nikolaev, H. Dai, P. Petit, J. Robert, C. Xu, Y.H. Lee, S.G. Kinm, A.G. Rinzler, D.T. Colbert, G.E. Scuseria, D. Tománek, J.E. Fisher, and R.E. Smalley, Science 273, 483 (1996)

\footnotetext{
${ }^{2}$ S. Iijima, Nature (London) 354, 56 (1991).

${ }^{3}$ W. Krätschmer, L.D. Lamb, K. Fostiropoulous, and D.R. Hoffman, Nature (London) 347, 354 (1990).

${ }^{4}$ S. Saito and A. Oshiyama, Phys. Rev. Lett. 66, 2637 (1991).
} 
${ }^{5}$ M. Posternak, A. Baldereschi, A.J. Freeman, E. Wimmer, and M. Weinert, Phys. Rev. Lett. 50, 761 (1983).

${ }^{6}$ M. Posternak, A. Baldereschi, A.J. Freeman, and E. Wimmer, Phys. Rev. Lett. 52, 863 (1984).

${ }^{7}$ S. Saito and A. Oshiyama, Phys. Rev. Lett. 71, 121 (1993).

${ }^{8}$ Y. Miyamoto, A. Rubio, X. Blase, M.L. Cohen, and S.G. Louie, Phys. Rev. Lett. 74, 2993 (1995).

${ }^{9}$ X. Blase, A. Rubio, S.G. Louie, and M.L. Cohen, Europhys. Lett. 28, 335 (1994).

${ }^{10}$ P. Hohenberg and W. Kohn, Phys. Rev. 136, B864 (1964).

${ }^{11}$ W. Kohn and L.J. Sham, Phys. Rev. 140, A1133 (1965).

${ }^{12}$ D.M. Ceperley and B.J. Alder, Phys. Rev. Lett. 45, 566 (1980).

${ }^{13}$ J.P. Perdew and A. Zunger, Phys. Rev. B 23, 5048 (1981).

${ }^{14}$ N. Troullier and J.L. Martins, Phys. Rev. B 43, 1993 (1991).

${ }^{15}$ L. Kleinman and D.M. Bylander, Phys. Rev. Lett. 48, 1425 (1982).

${ }^{16}$ O. Sugino and A. Oshiyama, Phys. Rev. Lett. 68, 1858 (1992).
${ }^{17}$ N. Hamada, S. Sawada, and A. Oshiyama, Phys. Rev. Lett. 68, 1579 (1992).

${ }^{18}$ R. Saito, M. Fujita, M.S. Dresselhaus, and G. Dresselhaus, Appl. Phys. Lett. 60, 2204 (1992).

${ }^{19}$ When we neglect the curvature of the nanotubes, the narrow-gap semiconducting tubes become metallic (Ref. 18). The tube index $(m, n)$ used in this paper is transformed from that used in our previous work $\left(n_{1}, n_{2}\right)$ (Ref. 17) by the following relation; $n_{1}$ $=m+n, n_{2}=n$.

${ }^{20}$ M. Saito and A. Oshiyama, Phys. Rev. B 48, 11804 (1993).

${ }^{21}$ R.S. Lee, H.J. Kim, J.E. Fisher, A. Thess, and R.E. Smalley, Nature (London) 388, 255 (1997).

${ }^{22}$ C. Bower, S. Suzuki, K. Tanigaki, and O. Zhou, Appl. Phys. A: Mater. Sci. Process. 67, 47 (1998).

${ }^{23}$ C. Bower, A. Kleinhammes, Y. Wu, and O. Zhou, Chem. Phys. Lett. 288, 481 (1998). 